\title{
Protein A Immunoadsorption combination with Immunosuppressive Therapy Improve Neuropsychiatric Systemic Lupus Erythematosus: A Case Report
}

Feng Wen ${ }^{1}$, Xiaoling $\mathrm{Wu}^{2}$, Ruyi $\mathrm{Liao}^{3}$, Zhonglin Feng ${ }^{4}$, Zhilian $\mathrm{Li}^{4}, \mathrm{Xia} \mathrm{Fu}^{3}$, Lei Fu ${ }^{3}$, Sijia $\mathrm{Li}^{3}$, Zhuo $\mathrm{Li}^{3}$, Sheng $\mathrm{Li}^{3}$, Wenjian Wang ${ }^{4}$, Biao Huang ${ }^{3}$, Chaosheng $\mathrm{He}^{5}$, Wei $\mathrm{Shi}^{5}$, Xinling Liang $^{5}$, and shuangxin liu ${ }^{6}$

${ }^{1}$ Guangdong Provincial People's Hospital, Guangdong Academy of Medical Sciences ${ }^{2}$ Guangdong Provincial People's Hospital, Guangdong Academy of Medical Sciences ${ }^{3}$ Guangdong Provincial People's Hospital, Guangdong Academy of Medical Sciences, ${ }^{4}$ Guangdong Provincial People's Hospital, Guangdong Academy of Medical Sciences, ${ }^{5}$ Guangdong Provincial People's Hospital, Guangdong Academy of Medical Sciences,

${ }^{6}$ Affiliation not available

May 7, 2020

\begin{abstract}
Neuropsychiatric systemic lupus erythematosus (NPSLE) is usually associated with a worse prognosis. However, there are few effective treatments for NPSLE. We described protein A immunoadsorption treatment for a patient with NPSLE. The patient's brain lesions was improved rapidly after two sessions of protein A immunoadsorption combination with immunosuppressive agent treatment.

Running title: Protein A Immunoadsorption and Systemic Lupus Erythematosus

Title: Protein A Immunoadsorption combination with Immunosuppressive Therapy Improve Neuropsychiatric Systemic Lupus Erythematosus: A Case Report

Feng Wen M. D. ${ }^{1, \#}$, Xiaoling Wu M. D. ${ }^{2,3, \#}$, Ruyi Liao M. D. ${ }^{1, \#}$, Zhonglin Feng M. D. ${ }^{1}$, Zhilian Li M. D. ${ }^{1}$, Xia Fu M. D. ${ }^{1}$, Lei Fu, M. D. ${ }^{1}$, Sijia Li M. D. ${ }^{1}$, Zhuo Li M. D. ${ }^{1}$, Sheng Li M. D. ${ }^{1}$, Wenjian Wang, M. D. ${ }^{1}$, Biao Huang M. D. ${ }^{2,3}$, Chaosheng He M. D. ${ }^{1}$, Shi Wei M. D. ${ }^{1}$,Xinling Liang M. D. ${ }^{1,2, \&}$, Shuangxin Liu M. D. ${ }^{1,2, \&}$

${ }^{1}$ Department of Nephrology, Guangdong Provincial People's Hospital, Guangdong Academy of Medical Sciences, Guangzhou, 510080, China.

${ }^{2}$ Medical School, South China University of Technology, Guangzhou, 510080, China.

${ }^{3}$ Department of Radiology, Guangdong Provincial People's Hospital, Guangdong Academy of Medical Sciences, Guangzhou, 510080, China.

\# The authors contributed equally to this work.

$\&$ The authors are co-correspondence authors.
\end{abstract}

Manuscript information: 49 words in Abstract and 1343 words in Main text 
1 Figure (1 black \& white figure) and 1 Table.

Correspondence authors

Xinling Liang, Department of Nephrology, Guangdong Provincial People's Hospital, Guangdong Academy of Medical Sciences, Guangzhou 510080, People's Republic of China

Phone: +8613808819770; Fax: (86)20-83827812-62027; E-mail:xinlingliang_ggh@163.com

Shuangxin Liu, Department of Nephrology, Guangdong Provincial People's Hospital, Guangdong Academy of Medical Sciences, Guangzhou, 510080, People's Republic of China

Phone: +8613543456446; Fax: (86)20-83827812-62027; E-mail:13543456446@163.com

Abstract

Neuropsychiatric systemic lupus erythematosus (NPSLE) is usually associated with a worse prognosis. However, there are few effective treatments for NPSLE. We described protein A immunoadsorption treatment for a patient with NPSLE. The patient's brain lesions was improved rapidly after two sessions of protein A immunoadsorption combination with immunosuppressive agent treatment.

Key Words: Protein A immunoadsorption, Neuropsychiatric systemic lupus erythematosus,

\section{Key Clinical Message}

We described firstly protein A immunoadsorption combination with immunosuppressive treatment improved rapidly a patient with NPSLE.

\section{INTRODUCTION}

Systemic lupus erythematosus (SLE) is a chronic autoimmune disease with a broad spectrum of clinical presentations [1]. The neuropsychiatric SLE (NPSLE) presents with heterogeneous symptoms, such as headache, cognitive impairment, memory loss, seizure and stroke which contributes considerably to morbidity and mortality. The development and deterioration of NPSLE are associated with the formation of a large number of auto-antibodies and circulating immune complexes in the body, which could cause severe brain damage and even life-threatening [2]. Inhibiting or removing pathogenic auto-antibodies could prevent their pathological outcomes [1]. Severe NPSLE should be treated with immunosuppressive and biological agents. However, some patients are not sensitive to these drugs. Protein A immunoadsorption (IAS) has been shown to improve disease activity and lower glucocorticoid dosages in lupus nephritis [3]. Braun and colleague reported that protein A immunoadsorption had effect on severe and therapy-resistant SLE, which suggested IAS as a possible option when other therapies were ineffective [4]. We presented a case of NPSLE, who received 3 sessions of IAS treatment after poor responses to immunosuppressive agents. The significant improvement of brain magnetic resonance imaging (MRI) manifestations have been firstly reported after the comprehensive treatments.

\section{CASE HISTORY}

A 20-year-old woman, who had fever and red rash on her face, and was admitted firstly on April 2016. Plasma antinuclear antibody (ANA), anti-dsDNA and proteinuria were increased. She was diagnosed as SLE and lupus nephropathy (type II) by kidney biopsy. Oral methylprednisolone (MP, 40mg daily) and hydroxychloroquine (HCQ, 100mg, twice daily) were prescribed. The rash fade and the proteinuria completely relieved after 2 months treatments. She regularly decreased the dosage of MP. On August 2018, when the MP was reduced to $4 \mathrm{mg}$ daily, the rash on face reappeared. Two months later, her right eye presented with blurred vision. The blood tests indicated an elevated of ANA titer to 1:1280, anti-dsDNA concentration to $74.5 \mathrm{IU} / \mathrm{ml}$. The blood IgG concentration was $18.1 \mathrm{~g} / \mathrm{L}$. The plasma C3 level slightly declined and proteinuria stayed normal. HCQ was stopped immediately and MP of $12 \mathrm{mg}$ daily was given. Although the 
rash soon improved, the patient had fever, and the visual impairment developed to both sides. The first cerebrospinal fluid (CSF) examination showed a normal tension of $170 \mathrm{mmH}_{2} \mathrm{O}$, with an increased protein concentration of $922 \mathrm{mg} / \mathrm{L}$, a slightly elevation of $\mathrm{IgG}$ level and leukocyte count. Pathogen examinations including bacterial, fungal cultures and variety of viral antibodies were all negative in the CSF and blood tests. In brain MRI scan, the axial fluid attenuated inversion recovery (FLAIR) sequence showed hyper-intense in the right basal ganglia and bilateral peri-ventricular white matter, without abnormal enhancement (Figure 1 A-C). Ophthalmic examinations suggested retinal vasculitis in both eyes. The patient was identified as NPSLE with a disease activity score of 19 (brain damage, visual impairment, rash, and fever). Intravenous MP (500mg daily for 3 days) and cyclophosphamide (200mg every other day) were prescribed. Prednisolone of $45 \mathrm{mg}$ daily was given subsequently.

However, the vision did not improve after intravenous MP combined with cyclophosphamide treatment. The second brain MRI scan showed that lesions of abnormal hyper-intense were increased in the right lateral temporal cortex, right thalamus bilateral basal ganglia, insular lobe external capsule, and peri-ventricular white matter on FLAIR images. Intravenous MP (500 $\mathrm{mg}$ daily for 3 days) were used again, and rituximab (500mg) was prescribed twice. Intrathecal injections of methotrexate and dexamethasone were performed weekly for a total of six times. Although the patient was treated with intensive therapy, the CSF examinations turned out to be a significantly increase of tension $\left(218 \mathrm{mmH}_{2} \mathrm{O}\right)$ and protein concentration $(2187 \mathrm{mg} / \mathrm{L})$, and area of brain lesions still expanded by MRI scan (Figure 1 D-F). The patient's consciousness become worse accompanied with dysphagia.

Because prednisolone and immunosuppressive agents had no effects on retinal vasculitis and consciousness, protein A IAS (KONCHEN Biotech. Co., Ltd., Guangzhou, China) was performed. Three protein A IAS sessions were executed. Each session ran 6 circles and total volume of plasma adsorption was $3600 \mathrm{ml}$. Her consciousness got better soon after the first session. After the second session, examination of CSF showed a normal tension of $165 \mathrm{mmH}_{2} \mathrm{O}$ and a decrease of protein concentration to $547 \mathrm{mg} / \mathrm{L}$. The brain lesions on MRI were found to be significantly reduced (Figure 1 G-I). Plasma IgG decreased significantly after each IAS sessions (Table 1). The protein A IAS was interrupted because of catheter related deep vein thrombosis after the third section. Two weeks later, the patient discharged with oral MP (40mg daily). Her consciousness and strength completely recovered after one month, but the vision has still not restored.

\section{DISCUSSION}

The patient became severe during the process of glucocorticoid reduction, and her brain was involved in damage. Although the changes of laboratory tests were mild, the neuropsychiatric symptoms and brain MRI were progressive. The symptoms became worse, even if intensive immunosuppressive and biological agents were prescribed. It was worthy of note that, the brain MRI was rapidly improved after two sessions of IAS were performed, together with intravenous cyclophosphamide and immunosuppressive therapy.

Glucocorticoid combination with intravenous pulse cyclophosphamide is the most classical strategy for SLE with major organs involved [5]. It's effective in many case, but not all situations. When common strategies are contraindicated or fail in life-threatened SLE, extracorporeal treatments are performed. Although plasma exchange could non-selectively remove the antibodies, it is reported to be not effective in prospective trail [6]. In contrast, IAS can reduce significantly the concentration of IgG in blood without loss of coagulation factors and plasma albumin. Stummvoll and colleague found that IAS improved significantly disease activity and lowered glucocorticoid dosages in SLE patients [3,7]. These patients showed benefits within 3 months of treatment and stabilization thereafter. IAS might be used as an optional treatment when other therapies are not effective in SLE [4].

NPSLE is associated with a worse prognosis, and brain MRI scan is recommended to evaluate brain lesions in patients [8]. The most frequent MRI findings are focal lesions in subcortical white matter, cortical atrophy, diffuse cortical grey matter changes, less brain and corpus callosum volumes in patients with NPSLE $[9,10]$. The patient showed abnormal hyper-intense in right basal ganglia at the beginning, similar to cerebral infarction. When the disease progressed, atrophy of the brain, diffuse white matter and multiple nuclear 
lesions were identified. The formation of these manifestations contribute to blood-brain barrier dysfunction, cerebrovascular disease, serum and CSF autoantibody-mediated injury [11]. Microinfarcts may be responsible to retinal vasculitis and basal ganglia lesions in this patient. The auto-antibodies deposition in the brain play an important role in the pathogenesis [12]. Some of them, such as antiphospholipid antibodies, are reported more likely to be elevated in NPSLE [13]. Besides, evidences suggest that these immunoglobulins and immune cells could leak into brain tissue and cause immune responses.

The ability of elimination of circulating complexes by protein A IAS was confirmed in several autoimmune diseases [14-16]. Braun and colleague reported the elimination kinetics of IgGs and circulating immune complexes in vivo during the treatment of severe SLE [17]. It showed that all IgG subclasses were removed from the patient's plasma. The half time of $\operatorname{IgG}$ elimination is 4.8 days during intermittent therapy. Protein A IAS decreases IgG levels in the blood, leads to a reduction IgG in cerebrospinal fluid, and then improves the clinical symptoms of NPSLE. The immunosuppressive treatment could reduce the recomposition and redistribution. Thus the treatment of adsorption could be better, when immunosuppressive agents are prescribed. Because of competition for protein A binding sites, intravenous immunoglobulin prevents the rapid decline of IgG in the patients, which is suggested to be avoided during protein A IAS treatment[17].

\section{CONCLUSION}

In summary, we firstly reported a case of NPSLE with severe brain damage, who was improved rapidly after protein A IAS combination with immunosuppressive treatment. Although IAS treatment is effective in the patient with NPSLE, we need to do more studies to verify the effectiveness of protein A immunoadsorption in future.

\section{Abbreviations}

SLE, systemic lupus erythematosus; NPSLE, neuropsychiatric systemic lupus erythematosus; IAS, immunoadsorption; MRI, magnetic resonance imaging; ANA, antinuclear antibody; MP, methylprednisolone; HCQ, hydroxychloroquine; CSF, cerebrospinal fluid; FLAIR, fluid attenuated inversion recovery.

\section{Acknowledgements}

We acknowledge Guobiao Hong and Li Song in the blood purification center of Guangdong Provincial People's Hospital for operating and providing medical care during the patient's protein A immunoadsorption treatments.

\section{Funding}

This study was supported by National Natural Science Foundation of China (81670656,81870508, funder SXL), China Postdoctoral Science Foundation (2017M612624, funder RYL),Natural Science Foundation of Guangdong Province (2014A030313544, funder CSH), Guangdong Science and Technology Fund (2014A020209001, funder CSH),Guangdong Medical Science and Technology Research Fund (A2017564, funder RYL), Guangzhou City Science and Technology Project (201707010009, funder SXL). These funding support part treatment fee.

\section{Availability of data and material s}

The clinical data regarding this case is stored in hospital medical records.

\section{Authors' contributions}

All authors have read and approved the manuscript.

FW and RYL collected the clinical data of the patient, performed the literature search and drafted the manuscript. XLW provided and evaluated the MR image and drafted part of the manuscript. ZLF and ZLL participated in the data collection. XF, SJL, ZL, SL, WJW, CSH, and WS established the diagnosis and agreed the patient's management plan. BH participated in preparation the results of the MR images. XLL and SXL supervised and drafted the manuscript. 


\section{Ethics approval and consent to participate}

Because it is a case report, this study was exempted from research ethics committee review (Ethics committee of Guangdong Provincial People's Hospital). The patient has gave consent for publication.

\section{Consent for publication}

Written informed consent was obtained from the patient for publication of this case report. A copy of the consent form is available for review and can be provided on request.

\section{Competing interests}

The authors declare no competing interests.

\section{References}

1. Tsokos GC. Systemic lupus erythematosus. N Engl J Med. 2011;365(22):2110-2121.

2. Duarte-Delgado NP, Vasquez G, Ortiz-Reyes BL. Blood-brain barrier disruption and neuroinflammation as pathophysiological mechanisms of the diffuse manifes tations of neuropsychiatric systemic lupus erythematosus. Autoimmun Rev. 2019;18(4):426-432.

3. Stummvoll GH, Aringer M, Smolen JS, et al. IgG immunoadsorption reduces systemic lupus erythematosus activity and proteinuria: a long term observational study. Ann Rheum Dis. 2005;64(7):10151021.

4. Braun N, Erley C, Klein R, et al. Immunoadsorption onto protein A induces remission in severe systemic lupus erythematosus. Nephrol Dial Transplant. 2000;15(9):1367-1372.

5. Illei GG, Austin HA, Crane M, et al. Combination therapy with pulse cyclopho sphamide plus pulse methylprednisolone improves long-term renal outcome without adding toxicity in patients with lupus nephritis. Ann Intern Med. 2001;135(4):248-257.

6. Lewis EJ, Hunsicker LG, Lan SP, et al. A controlled trial of plasmapheresis th erapy in severe lupus nephritis. The Lupus Nephritis Collaborative Study Group. N Engl J Med. 1992;326(21):1373-1379.

7. Stummvoll GH, Aringer M, Jansen M, et al. Immunoadsorption (IAS) as a rescue therapy in SLE: considerations on safety and efficacy. Wien Klin Wochenschr. 2004;116(21-22):716-724.

8. Zirkzee EJ, Huizinga TW, Bollen EL, et al. Mortality in neuropsychiatric system mic lupus erythematosus (NPSLE). Lupus. 2014;23(1):31-38.

9. Postal M, Lapa AT, Reis F, et al. Magnetic resonance imaging in neuropsychiatric systemic lupus erythematosus: current state of the art and novel approaches. Lupus. 2017;26(5):517-521.

10. Jeltsch-David H, Muller S. Neuropsychiatric systemic lupus erythematosus: path ogenesis and biomarkers. Nat Rev Neurol. 2014;10(10):579-596.

11. Hanly JG. Diagnosis and management of neuropsychiatric SLE. Nat Rev Rheumatol. 2014;10(6):338347.

12. Yaniv G, Twig G, Shor DB, et al. A volcanic explosion of autoantibodies in systemic lupus erythematosus: a diversity of 180 different antibodies found in SLE patients. Autoimmun Rev. 2015;14(1):75-79.

13. Ho RC, Thiaghu C, Ong H, et al. A meta-analysis of serum and cerebrospinal fluid autoantibodies in neuropsychiatric systemic lupus erythematosus. Autoi- mmun Rev. 2016;15(2):124-138.

14. Antozzi C, Berta E, Confalonieri P, et al. Protein-A immunoadsorption in immunosuppression-resistant myasthenia gravis. Lancet. 1994;343(8889):124.

15. Griveas I, Sourgounis A, Visvardis G, et al. Immunoadsorption in lupus myocarditis. Ther Apher Dial. 2004;8(4):281-285.

16. Maillard N, Absi L, Claisse G, et al. Protein A-Based Immunoadsorption Is More Efficient Than Conventional Plasma Exchange to Remove Circulating Anti-HLA Antibodies. Blood Purif. 2015;40(2):167172 .

17. Braun N, Gutenberger S, Erley C M, et al. Immunoglobulin and circulating immune complex kinetics during immunoadsorption onto protein A sepharose. Transfus Sci. 1998;19 Suppl:25-31. 


\section{Legends}

Figure 1. The changes of brain lesions in patient's MR scan during treatment.

The abnormal signals could be found in axial fluid-attenuated inversion recovery (FLAIR) sequences. Patients' first MRI showed diffuse and symmetric hyper-intense areas in the bilateral peri-ventricular white matter, basal ganglia and insula (A-C). Compared with first MR scan, the area of brain lesions expanded obviously before protein A immunoadsorptions treatment (D-F). After two sessions of protein A immunoadsorptions, FLAIR images of MRI decreased significantly area of lesions (G-I).

Table 1. Changes of blood immune indicators before and after immunoadsorption sessions.
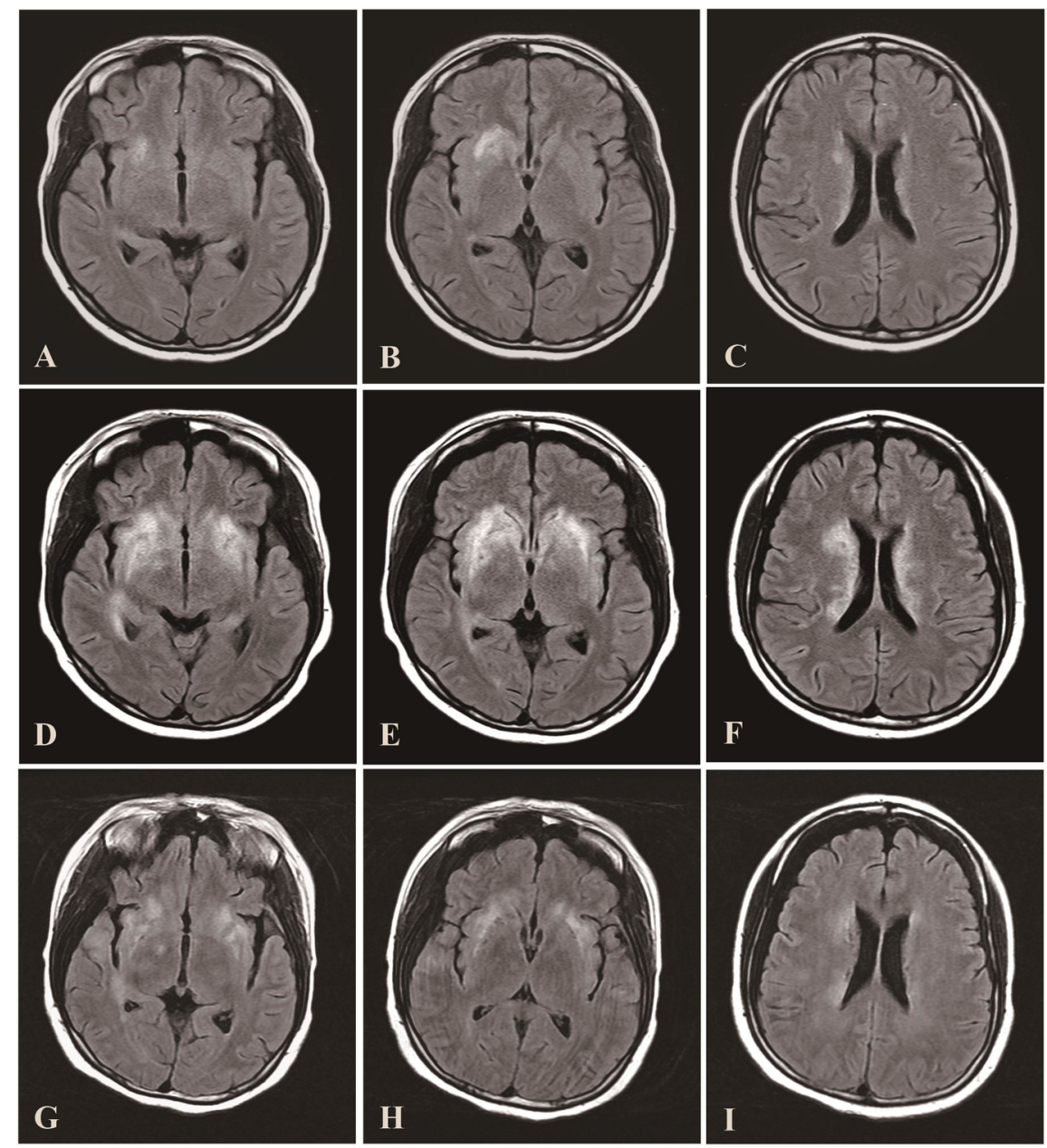

\section{Hosted file}

Table 1.docx available at https://authorea.com/users/319232/articles/448978-protein-aimmunoadsorption-combination-with-immunosuppressive-therapy-improve-neuropsychiatricsystemic-lupus-erythematosus-a-case-report 